\title{
Injuries in Children with Autism Spectrum Disorder: Study to Explore Early Development (SEED)
}

\author{
Carolyn DiGuiseppi ${ }^{1}$, Susan E. Levy ${ }^{2}$, Katherine R. Sabourin ${ }^{1}$, Gnakub N. Soke ${ }^{1,3}$, Steven \\ Rosenberg $^{4}$, Li-Ching Lee ${ }^{5}$, Eric Moody ${ }^{4}$, and Laura A. Schieve ${ }^{3}$ \\ ${ }^{1}$ Department of Epidemiology, Colorado School of Public Health, University of Colorado Anschutz \\ Medical Campus, 13001 E. 17th Place, Campus Box B119, Aurora, CO 80045, USA \\ ${ }^{2}$ The Children's Hospital of Philadelphia at University of Pennsylvania, Philadelphia, PA 19104, \\ USA
}

${ }^{3}$ National Center on Birth Defects and Developmental Disabilities, Centers for Disease Control and Prevention, Atlanta, GA 30341, USA

${ }^{4}$ Department of Psychiatry, School of Medicine, University of Colorado Anschutz Medical Campus, Aurora, CO 80045, USA

${ }^{5}$ Department of Epidemiology, Johns Hopkins Bloomberg School of Public Health, Baltimore, MD 21295, USA

\section{Abstract \\ This study examined caregiver-reported medically-attended injuries among 30-68 month old children with autism spectrum disorder (ASD) compared to general population (POP) and non- ASD developmental disorders (DD) controls in the Study to Explore Early Development. Injuries were common in ASD cases (32.3\%) as well as POP (30.2\%) and DD (27.8\%) controls; most resulted in an emergency visit or hospitalization. After accounting for sociodemographic, health, IQ and behavior differences, odds of injury in ASD cases were significantly higher than DD controls but similar to POP controls. Attention problems mediated the relationships. Clinicians}

\footnotetext{
Correspondence to: Carolyn DiGuiseppi.

Disclaimer: The findings and conclusions in this report are those of the authors and do not necessarily represent the official position of the Centers for Disease Control and Prevention.

Author Contributions: CD, SEL, SR, L-CL and LAS contributed to conceptualization and design of the SEED study and oversaw acquisition of data. EM and GNS contributed substantially to data acquisition. CD, SEL, and KRS coded injury data. CD, KRS and GNS designed the statistical analyses, and KRS and GNS performed statistical analyses. CD and KRS drafted the manuscript. All authors participated in the interpretation of data, critically revised the manuscript for important intellectual content, and approved the final version to be published.

Compliance with Ethical Standards

Conflict of interest: The author declares that they have no conflict of interest.

Ethical Approval: This study was approved by Institutional Review Board (IRB)-C, CDC Human Research Protection Office; Kaiser Foundation Research Institute (KFRI) Kaiser Permanente Northern California IRB, Colorado Multiple IRB, Emory University IRB, Georgia Department of Public Health IRB, Maryland Department of Health and Mental Hygiene IRB, Johns Hopkins Bloomberg School of Public Health Review Board, University of North Carolina IRB and Office of Human Research Ethics, IRB of The Children's Hospital of Philadelphia, and IRB of the University of Pennsylvania. All procedures performed in this study were in accordance with the ethical standards of the institutional and national research committees and with the 1964 Helsinki declaration and its later amendments or comparable ethical standards.

Informed Consent: Informed written consent was obtained from all participating families included in this study.
} 
caring for children with both ASD and attention problems should consider providing targeted safety advice. Differences in injury risk between children with ASD vs. other developmental disorders need further study.

\section{Keywords}

Injuries; Autism spectrum disorder; Developmental delays and disorders; Epidemiology; Prevalence

\section{Introduction}

Among US children aged 3-5 years, injuries are the leading cause of death, at a rate of 7.1 deaths/100,000 population in 2015 (Centers for Disease Control and Prevention 2016). Injuries and poisonings are also a common cause of emergency room visits at this age. An estimated 1.1 million (9.3\%) children aged 3-5 years had an injury-related emergency department (ED) visit in 2015, resulting in more than 18,000 hospitalizations (1.5/1000) (Centers for Disease Control and Prevention 2016); the most common causes of these injuries were unintentional falls, being struck by or striking against a person or an object, bites and stings, foreign bodies (e.g., choked on food, swallowed battery), and cutting or piercing. Among US children aged 0-14 years injured in 2013, the estimated lifetime medical and work-loss costs from emergency department-treated nonfatal injuries were $\$ 59.1$ billion and from fatal injuries were $\$ 7.8$ billion (Florence et al. 2015).

Numerous risk and protective factors for injuries to children have been reported, including socio-demographic factors, maternal psychiatric disorders, and child psychological and behavioral problems (Bijur et al. 1992; Borse et al. 2008; Chakravarthy et al. 2010; Haynes et al. 2003; Hong et al. 2010; Mytton et al. 2009; Oliver and Kohen 2010; Phelan et al. 2007; Schwebel and Brezausek 2008; Schwebel and Gaines 2007). A number of these risk factors for injury occur at higher rates in children with autism spectrum disorder (ASD) and their families, which could result in higher injury rates in these children. Such factors include male predominance; child psychological and behavioral problems including hyperactivity, anxiety, aggressive behavior, cognitive delays affecting mental processing or causal reasoning, and sensory deficits; and maternal psychopathology (e.g., anxiety, depression) (Daniels et al. 2008; Hodge et al. 2011; Newschaffer et al. 2007).

Several studies in recent years have examined injury risk among individuals with ASD. In a large, nationally representative sample of children aged 3-5 years, Lee et al. (2008) reported a significantly higher risk of parent-reported injury requiring medical attention among children with autism compared to unaffected controls, after accounting for sociodemographic differences (risk ratio $(\mathrm{RR})=2.15 ; 95 \%$ confidence interval $(\mathrm{CI}): 1.00$, 4.60) (Lee et al. 2008). Another large, population-based study enrolled children aged 1 to < 18 years covered by Medicaid (a government insurance program for families with insufficient resources to pay for health care) (McDermott et al. 2008). In this study, the risk of injury requiring emergency or hospital treatment was modestly higher among children with autism or other pervasive developmental disorder (PDD) compared to children without any PDD (RR 1.20; 95\% CI 1.04, 1.39) after controlling for age and gender. The injury risk 
for children with both autism/other PDD and intellectual disability (ID) was similar to unaffected children (RR 1.1; 95\% CI 0.94, 1.30), while the risk was higher for children with autism/other PDD without ID (RR 1.75; 95\% CI 1.28, 2.40). Vohra et al. (2016), in a study of adults aged 22-64 years seen in the emergency department (ED), found a significantly higher odds of the visit being due to an injury in those with ASD compared to those without ASD (OR 1.10; 95\% CI 1.04, 1.16) after adjusting for sociodemographic differences, hospital location and patient disposition (Vohra et al. 2016). Further, using multiple causeof-death data files from the National Vital Statistics System, 1999-2014, Guan and Li (2017) identified a nearly three-fold higher age-adjusted proportionate mortality ratio (PMR) for deaths attributed to injury in individuals with autism compared to the general population after accounting for age and gender differences (PMR 2.93; 95\% CI 2.64, 3.24), with the highest PMRs for drowning, asphyxiation and suffocation (Guan and Li 2017).

Not all studies have reported higher risk of injury associated with ASD, however. Kalb et al. (2016), using data from the 2008 Nationwide ED Sample, a national all-payer ED database, reported that the odds of an injury-related ED visit were 48\% lower among children aged 317 years with ASD without ID compared to a control group without ASD or ID, after accounting for sociodemographic differences (OR 0.52; 95\% CI 0.50, 0.54) (Kalb et al. 2016). Notably, ED visits in the ASD group were 5 times more likely to result from a selfinflicted injury than ED visits in the control group (OR 5.4; 95\% CI 4.2, 6.9). Another large study reported no significant association between the presence of special needs likely to affect behavior, including ASD, and risk of motor vehicle crash injury (OR 1.26; 95\% CI $0.71,2.25$ ), after adjusting for demographic factors (Huang et al. 2009). In that study, children with ASD had the lowest injury risk (0.54\%) and those with Attention Deficit/ Hyperactivity Disorder (ADHD) and/or externalizing disorders had the highest risk (3.85\%), compared to unaffected controls $(1.54 \%)$.

Differing results among these studies may be due in part to differences in the range of ages included, as well as to differences in distributions of sociodemographic characteristics, as only half of the studies cited adjusted for socioeconomic status in their analyses (Kalb et al. 2016; Lee et al. 2008; Vohra et al. 2016). Further, none of these studies controlled for differences in co-occurring conditions that may have influenced injury risk. We found one study of injury risk in ASD that accounted for potential differences in both clinical as well as sociodemographic factors. Jain et al. (2014) examined national insurance claims data for persons aged $<21$ years, controlling for diverse co-occurring conditions, such as attentiondeficit disorders, anxiety, and intellectual disability, as well as for sociodemographic factors (Jain et al. 2014). This study reported that those with ASD were at lower overall risk of injury [adjusted Hazard Ratio (aHR) 0.89; 95\% CI 0.87, 0.91], although injury risk was increased in the subset of children aged 3-5 years with ASD compared to unaffected controls (aHR 1.28; 95\% CI 1.23, 1.34).

SEED, a large multi-site, community-based case-control study, offers important advantages for further investigation of this problem (Schendel et al. 2012). In particular, SEED collects comprehensive data on numerous clinical and behavioral covariates known to be or potentially associated with injury risk. In addition, unlike studies based on insurance claims, SEED identifies and enrolls children not previously diagnosed with autism, who may lack 
health insurance or access to care (Mandell et al. 2009; Schendel et al. 2012). Further, SEED provides both developmentally-disabled and typically-developing study groups for comparison, enabling differentiation of the effects specific to ASD from those more generally resulting from neurodevelopmental disorders.

This study will describe injuries in young children with ASD, determine injury risks in children with ASD compared to children who are typically developing and to children with developmental delays and disorders, and examine whether these risks are modified or mediated by intellectual functioning or behavioral diagnoses or problems.

\section{Methods}

\section{Study Design and Setting}

SEED is a multi-site case-control study, for which the methods have been previously detailed (Schendel et al. 2012). Six sites (California, Colorado, Georgia, Maryland, North Carolina, and Pennsylvania) were included in SEED Phase 1 and in this analysis. Cases include children clinically evaluated for and diagnosed with autism spectrum disorder (ASD). The study included two control groups, children from the general population (POP) and children with non-ASD developmental delays/disorders (DD) such as language delay, motor delay, hearing problems, or sensory integration disorder.

\section{Participants}

Children were eligible for Phase 1 study enrollment if they were born between September 1, 2003 and August 31, 2006 in a study catchment area and lived in the same area at first contact. Because caregivers were a major source of information for the child's past behaviors, medical history, and exposures, a child was eligible for inclusion only if at the time of recruitment they resided with their caregiver aged at least 18 years who had taken care of the child continuously since they were 6 months of age. The caregivers were required to speak English or, at two study sites (California and Colorado), English or Spanish. The demographics and characteristics of the different study catchment sites have been previously described (DiGuiseppi et al. 2016; Schendel et al. 2012). Children were enrolled so as to be between 30.0 and 68.9 months old at the time of their clinical evaluation. This age range was chosen in order to limit recall bias for events in pregnancy and early life as much as possible, while still allowing diagnostic accuracy for ASD and maintaining the appropriate age range for validated study instruments. Children were recruited for the ASD and DD groups from educational and clinical settings that serve children with ASD and other developmental delays and disorders. Children from the general population were recruited from randomly sampled birth certificates. Families were sent an introductory letter followed by a phone call to assess eligibility.

\section{Data Collection and Study Group Classification}

The Social Communication Questionnaire (SCQ) was administered to parents of eligible children (Rutter et al. 2003). The SCQ was used to identify any children with possible undiagnosed ASD, defined as a score $\geq 11$ (Allen et al. 2007; Lee et al. 2007). Enrolled families completed interviews and forms about the child and parents, and enrolled children 
received clinical developmental assessments including the Mullen Scales of Early Learning (MSEL) Early Learning Composite (ELC) to assess cognitive functioning (Mullen 1995).

The process for classifying children into final groups has been previously described (Wiggins et al. 2015b). Children at risk for ASD (SCQ score $\geq 11$ at enrollment, previous ASD diagnosis, or observed ASD symptoms during the MSEL, regardless of source population) were given additional clinical developmental assessments, including the Autism Diagnostic Observation-Schedule (ADOS) (Lord et al. 1999) and the Autism Diagnostic Interview-Revised (ADI-R) (Gotham et al. 2007). Among children given the additional assessments, those meeting the cutoff scores on these instruments were classified as ASD (Schendel et al. 2012; Wiggins et al. 2015b), while those not meeting the cutoff scores were classified as DD with ASD characteristics. Among children classified as ASD, core symptom severity was assessed using the ADOS calibrated severity score (ADOS CSS) (Gotham et al. 2009), a continuous variable ranging from 1 (minimal symptoms) to 10 (high degree of symptoms) that can be compared across ages and levels of ability. Children with a prior diagnosed developmental condition who were not at risk for ASD were classified as DD without ASD characteristics. Children recruited from the birth certificate sample who were not at risk for ASD (as defined above) were classified as POP. Only children with a final classification of ASD, DD without ASD characteristics or POP, who completed a clinic visit, were included in this analysis.

Each parent or other caregiver completed a telephone (or sometimes in-person) interview about family, child, and household characteristics, health conditions, and behaviors; $99 \%$ of interviewees were the child's mother. Caregivers also completed the Child Behavior Checklist (CBCL) (Achen-bach 1992) for behavioral characteristics.

The primary outcome, i.e., ever had any injury that required medical attention, was collected as part of the caregiver interview, which asked about a child's previous injuries that required medical attention and, for each injury, whether it had resulted in an emergency department visit or hospitalization. In addition, a free text description ("what was the injury?") was collected. Three investigators (CD, SL, KS) independently categorized each free text injury description by mechanism, region, and nature of injury, using categories developed by the National Center for Health Statistics (Fingerhut and Warner 2006; National Center for Health Statistics 2002). In the case of any discrepant categorization, the case was discussed and a consensus was reached. Parent-reported 'injuries' that were agreed by all three investigators not to represent an acute physical injury (e.g., 'fever', 'diarrhea') $(n=72)$ were not counted as medically-attended injuries, resulting in 44 children initially coded as having had at least one injury being recoded as never having had an injury.

Sociodemographic factors included as potential confounding variables included child sex (Male, Female) and age at enrollment (mean); maternal race/ethnicity (Non-Hispanic White, Non-Hispanic Black, Hispanic, Non-Hispanic Other), maternal education (Less than a bachelor's, bachelor's, or graduate or higher degree), maternal age at child's birth (mean), maternal birthplace (US, Other), primary language spoken at home (English, Spanish, Other), household income $(<\$ 50,000, \$ 50,000-\$ 90,000,>\$ 90,000)$, number of children living in the home $(1,2,3,4+)$ and number of people living in the home $(2,3,4,5+)$. 
Sociodemographic variables were missing in $<1 \%$ of participants, except household income, which was missing in 3\%. Child health conditions examined included maternal report of physician-diagnosed seizure disorder/epilepsy, ADHD, and 'behavior problems,' (missing ranged from $2.6 \%$ for seizure disorder to $5.7 \%$ for behavior problems). Child cognitive ability (Mullen ELC Standard Score) and externalizing behavior, attention problems and attention deficit/hyperactivity problems (CBCL) were also examined, as continuous variables. Maternal history of physician-diagnosed neurodevelopmental condition, depression, and any psychiatric condition were each examined (missing in 5.3, 6.3 and $5.3 \%$, respectively).

\section{Analysis Plan}

Associations between ASD and having at least one medically-treated injury ("any injury") and at least one injury requiring an emergency room visit or hospitalization ("serious injury”) were examined using mixed-effects logistic regression models, with a random intercept for site, to account for differences in recruitment populations by study site. Children with ASD were compared separately to the POP and DD control groups. A base model specified a priori included adjustment for child sex and maternal race/ethnicity and education. Other sociodemographic variables were then assessed as potential confounders to create a sociodemographic-adjusted model. Remaining variables were assessed as potential confounders using this adjusted model. Potential confounders were any variables associated with both outcome and exposure at p-value $<0.2$, using Chi square or t-test based on the Satterthwaite method because of unequal variance between groups. Variables were included as covariates in the final adjusted model if they either changed the regression estimate by > $10 \%$ or were statistically significant after addition to the model and after addition of other significant covariates. Stratified analyses were also conducted, examining injury risk in children with ASD vs. DD according to the ELC Standard Score (impaired [< 70] vs. not impaired 270 ) and CBCL-T scores for attention problems, externalizing behaviors and attention deficit/hyperactivity problems (clinical [ 270$]$ vs. borderline/normal $[<70]$ ). Adjusted models were created using the same covariates included in the un-stratified analysis. In exploratory analyses restricted to children with ASD, the relationships between ADOS calibrated severity score and risks of any injury and any serious injury were examined using similar methods to those described above. All results are reported as odds ratios. The conventional alpha level of 0.05 was used for testing statistical significance. All analyses were completed using SAS 9.3.

\section{Results}

Of 2295 children who completed a clinic visit and received a study group classification of ASD, Developmental Delays/Disorders without ASD characteristics (DD) or Population Control (POP), 43 (1.9\%) were excluded because the caregiver failed to complete the history of postnatal injuries. A total of 693 children with ASD, 676 DD children, and 883 POP children were included. Sociodemographic characteristics and child and maternal conditions in the three study groups are shown in Table 1. 
Injuries were most common among children with ASD (32.3\%) followed by POP (30.2\%) and DD (27.8\%); serious injuries showed a similar pattern (26.6, 24.7 and 23.4\% among children in the ASD, DD and POP groups, respectively) (Table 2). More than $80 \%$ of injured children in each group had only one reported injury and fewer than 5\% of children in any group had three or more injuries. Patterns of reported injuries and reported serious injuries were similar in all three groups (Table 2). The most common nature of injury specified was open wound, followed by fracture, which together accounted for about half of injuries in each group. Nature of injury was unspecified for about a quarter of all injuries in the three groups. About half of total reported injuries in all three groups occurred to the head, with upper extremity accounting for at least one-fifth of injuries in each group. Where specified, falls were the most common mechanism of reported injury, accounting for 55.5, 54.1 and $53.8 \%$ of specified injury mechanisms among ASD, DD and POP children, respectively, followed by 'struck by or against an object or person' and 'other specified, classifiable injury' in all three groups, which accounted for $25-30 \%$ of the remaining specified injuries in each group. However, at least half of injury mechanisms in each of the three groups were not specified in the free text descriptions.

In unadjusted analyses, there was no association between ASD case status and having any medically-attended injury or any serious injury, when compared to the POP group (Table 3). Adjustment for sociodemographic factors did not substantially influence these effect estimates. Neither self-reported maternal diagnosis of depression nor of any psychiatric condition confounded these estimates. Accounting for differences between groups in having a prior diagnosis of ADHD attenuated the association of ASD with any injury. There was little evidence of an association between ASD and any serious injury in unadjusted analyses or after adjustment for sociodemographic differences between groups. Similar to results for any injury, the association with serious injury was attenuated by inclusion of attention problems (from the CBCL) in the model. The association was strengthened by inclusion of child cognitive ability (MSEL ELC Standard Score), but remained small and not statistically significant.

When compared to the DD group, there was a modest, non-significant association between ASD and any medically-attended injury and any serious injury in crude analyses (Table 3). After adjustment for differences in sociodemographic characteristics, a significantly increased odds of any injury with ASD was observed (Table 3). Maternal depression did not confound this relationship, nor was there evidence of mediation by child IQ, behavior, or prior diagnosis of ADHD. There was a weak, non-significant association between ASD and serious injury in both unadjusted analysis and after adjusting for sociodemographic characteristics. This association was attenuated when the model accounted for differences between groups in attention problems.

In models adjusted for sociodemographic characteristics, the odds of any injury or any serious injury between children in the ASD and DD groups were similar when stratified by cognitive ability (impaired vs. not impaired) and by attention problems (clinical vs. borderline/normal). The data suggested the possibility that the association between any injury or any serious injury and ASD, when compared to DD, may vary according to the presence of externalizing behaviors or attention deficit/hyperactivity problems, with a small, 
positive association among those with borderline/normal scores for externalizing behavior or attention deficit/hyperactivity problems, and small negative associations among those with clinical scores for either of these, although the interaction terms were not statistically significant in any models.

Among children with ASD, there was no significant association between core symptom severity and risk of any injury in the unadjusted model (OR $0.95 ; 95 \%$ CI $0.86,1.05$ ) or in the base model adjusted for child sex and maternal race/ethnicity and education (OR 0.93; $95 \%$ CI $0.84,1.04)$. Similarly, there was no association of core symptom severity with risk of serious injury either in the unadjusted model (OR $0.99 ; 95 \%$ CI $0.89,1.11$ ) or in the sociodemographically-adjusted based model (OR $0.98 ; 95 \%$ CI $0.88,1.09$ ). Other sociodemographic variables assessed as potential confounders did not influence these effect estimates.

\section{Discussion}

This study found little evidence to suggest that young children with ASD are more likely to have a medically-attended injury, or an injury severe enough to result in an emergency department visit or hospitalization, compared to typically developing children sampled from the general population, after accounting for differences between groups in sociodemographic characteristics, maternal psychiatric conditions and child health conditions, cognitive ability, prior behavioral diagnoses and current behavioral problems. Similarly, the likelihood of a serious injury did not differ between children with ASD and children with non-ASD developmental delays and disorders after accounting for these factors. However, children with ASD had a small but statistically significant increased odds of having ever had a medically-attended injury compared to children with non-ASD developmental delays and disorders, after accounting for sociodemographic differences between groups. As with comparisons to population controls, maternal psychiatric conditions and child health conditions, cognitive ability, prior behavioral diagnoses, and current behavioral problems did not substantially influence this relationship.

Our finding of no association between ASD and injuries relative to the general population is consistent with findings from several other recent studies (Huang et al. 2009; Kalb et al. 2016), but contrasts with two large, nationally representative samples of children aged 3-5 years, both of which found a significantly higher injury risk among children with ASD compared to unaffected controls (Jain et al. 2014; Lee et al. 2008). Lee et al. (2008) found a two-fold higher odds of injury with autism compared to controls, but unlike our study, relied on parent-reported diagnosis of autism and did not adjust for co-occurring conditions or child behavior (Lee et al. 2008), which may at least partly explain differences in our findings. While parent-reported diagnosis of autism has been shown to be reliable (Centers for Disease Control and Prevention 2006), SEED used a comprehensive approach that identified many young children with ASD who had not yet received a diagnosis. Our effect estimate for children with ASD vs. population controls (aOR 1.2) was only slightly smaller in magnitude than the significant association reported by Jain et al. (2014) for children in the same 3-5 year age group (HR 1.28; 95\% CI 1.23, 1.34) (Jain et al. 2014), which may be in part related to higher power in the latter study. Unlike Jain et al., however, we were able to 
examine and adjust for differences in behavioral problems based on standardized instruments, which attenuated the observed associations between ASD and injury in our study and may help explain our smaller effect estimates.

The small but statistically significant increased odds of having ever had a medically-attended injury among children with ASD compared to children with non-ASD developmental delays and disorders was not explained by sociodemographic or other differences between groups.

A few previous studies have directly compared injury risk in individuals with ASD vs. those with other developmental disabilities, although none have reported results for children of preschool age. Kalb et al. (2016) found that children aged 3-17 years with ASD had a 1.5 times higher odds of injury than same-aged children with ID, after adjustment for sociodemographic differences (Kalb et al. 2016). A study of high school sports injuries reported a nearly five-fold higher risk in teens with autism compared to those with ID, after adjustment for potential confounders (Ramirez et al. 2009). On the other hand, in a cohort study of adults with intellectual disabilities, Finlayson et al. (2010) (Finlayson et al. 2010) reported an 85\% lower odds of injuries among those with autism than those without, although chance could not be excluded. In addition, several studies have reported lower risks of injury relative to non-affected controls among children with ASD than among children with ADD/ADHD or cognitive disorders when results were stratified by type of disability (Huang et al. 2009; Lee et al. 2008). It is possible that children in our DD group had more impairments in gross motor development, which we did not specifically assess in SEED but which have been shown by Myhre et al. (2012) to significantly decrease the risk of injury (OR 0.65, 95\% CI 0.42, 0.99) (Myhre et al. 2012). However, parent-reported motor delay was less common in our DD control group than in the ASD case group (Wiggins et al. 2015a). Another possibility is that parental perception of risk or parental supervision may differ among parents of children with ASD compared to parents of children with other nonASD developmental disorders. Since adequate adult supervision is necessary to protect young children from injury (Schwebel and Gaines 2007), any such differences could affect injury risk. It is also possible that the threshold to seek care, which would directly affect whether an injury resulted in medical attention (and was therefore reported as an outcome), may vary depending on child developmental condition. If parents of children with ASD were more likely to seek care for an injury event than parents of children with non-ASD developmental disorders, this could have resulted in a higher apparent injury risk in the ASD compared to the DD group. Lastly, our DD group comprised a wide variety of disorders that may have varying injury risks, which could have influenced our results.

As described in the introduction, this study has a number of strengths, including use of research-reliable administration of standardized instruments to evaluate and classify children with ASD, inclusion of children who had not previously received a medical diagnosis of autism (perhaps reflecting lack of access to care), collection of comprehensive data that enabled us to examine numerous important covariates known to be or potentially associated with injury risk, and inclusion of both a developmentally disabled and a typically-developing study group (Schendel et al. 2012). There were also several potential limitations to this study. The primary outcome was maternal recall of any medically attended injury in the child's lifetime (i.e., from birth to 3-5 years of age, depending on time of enrollment). Previous research has shown that maternal recall of medically-attended injuries in the past 
year among their children aged $<6$ years declines substantially with time (Cummings et al. 2005). Further, the maternal interview did not specifically ask about poisonings, which may not be perceived as injuries by some parents. Thus, the estimates reported here for lifetime injury occurrence are likely to substantially underestimate the true risk of injury among children with ASD as well as among control children. If this recall were differential between groups, the effect estimates may have been biased. Unfortunately, while population-based data for injuries in US children exist, they are not directly comparable to the data collected for SEED. The National Health Interview Survey estimates an age-adjusted annualized rate of injury episodes in children aged < 15 years of $11.2 \%$ (Chen et al. 2009), while the National Electronic Injury Surveillance System (NEISS), which collects injury visit data in a nationally representative sample of EDs, reports an annual nonfatal injury rate of $10.0 \%$ for children aged 3-5 years (Centers for Disease Control and Prevention 2016). In our sample, parents of children aged 3-5 years reported a lifetime prevalence of any injuries to be $30.2 \%$, and of injuries requiring an ED visit or hospitalization to be $24.7 \%$. Since injury rates in children vary substantially by age, we cannot reliably estimate an annualized injury rate for the SEED sample for comparison purposes. In addition, NEISS data are reported by injury rather than by child, hence a child with multiple injuries in a given year may be counted multiple times in NEISS, making comparisons to our data problematic. Still, our rates of 30.2 and $24.7 \%$ reported over an average period of approximately 4 years does not appear to be exceptionally high compared to national data. It is also possible that parents were less likely to report self-inflicted injuries, which have been shown to account for a slightly greater proportion of injury-related ED visits in children aged 3-17 years with ASD than without ASD (2.4 vs. 1.0\%, respectively) (Kalb et al. 2016), potentially biasing our results toward the null. However, the children studied in Kalb et al. were substantially older than in our study, which may have increased the likelihood of self-inflicted injuries being serious enough to result in an ED visit. We were unable to investigate this issue in the current analysis, as intentionality was not collected in the caregiver interview. The previously described relationship between maternal depression or anxiety and injury risk has been attributed to factors such as inadequate supervision and environmental safeguarding (Schwebel and Brezausek 2008; Schwebel and Gaines 2007), but could potentially also be influenced by biased reporting of injuries. Biased reporting of various child psychological traits and symptoms in mothers with psychopathology has been documented (e.g., in Rubenstein et al. 2017). However, we found no evidence that maternal psychiatric illness confounded the relationship between injury risk and ASD. In the free text response for injury description, parents typically reported the body region injured (ranging from 88 to $92 \%$ reported in the three study groups) and the nature of injury (72-78\% specified), but not usually the mechanism (41-50\% specified). Hence we were unable to examine whether the mechanism of injury differed between groups. Nevertheless, the most common specified mechanisms of injury were the same in all three groups, suggesting against important differences in mechanisms between groups. As described previously (DiGuiseppi et al. 2016), mothers of minority race, Hispanic ethnicity and low education were underrepresented among families enrolled in the POP group compared to the birth cohort, which may have resulted from SEED's relatively low recruitment contact rate (Schendel et al. 2012). Low response rates may increase the potential for biased measures of association if individuals in the ASD or comparison groups with certain sociodemographic 
characteristics or exposures responded disproportionately to study invitations. However, adjustment for sociodemographic differences had little effect on the findings.

Our findings suggest that previous reports of increased injury risk in this age group compared to general population controls may be explained, in part, by other differences between groups, in particular attention problems, that were not taken into account. Prior research has found an increased risk of injuries associated with parent-reported attention problems or diagnosis of ADHD (Myhre et al. 2012; Schwebel and Gaines 2007), which may result directly from the behavioral symptoms (e.g., impulsive behavior, inattention) or indirectly due to their effect on the parent (e.g., mental distress) or the parent-child relationship, which may in turn affect the quality of supervision (Schwebel and Gaines 2007). Some $40 \%$ of children with ASD have clinically significant ADHD symptoms and those with such symptoms have greater impairment in adaptive functioning compared to those without (Sikora et al. 2012). Our findings suggest that clinicians caring for children with ASD consider assessing the presence of attention problems and providing both targeted safety advice as well as support for the parent where such problems are identified. Further research is needed to explain differences in injury risk between children with ASD and children with other non-ASD developmental disorders, preferably involving longitudinal follow-up, shorter periods of recall, detailed information on the mechanism and intentionality of the injury, and detailed examination of parental conditions, perceptions and behaviors that may influence child injury risk. Finally, the absence of differences in injury risk among children with ASD compared to population controls aged 3-5 years might reflect higher levels of supervision typically provided to all children in this age group, regardless of their developmental functioning. Further exploration of injury risk in school-aged children and teens with ASD, where there is typically less supervision, may therefore be warranted.

\section{Acknowledgments}

This research is supported by the Centers for Disease Control and Prevention (CDC), Centers for Autism and Developmental Disabilities Research, Study to Explore Early Development through six cooperative agreements: Cooperative Agreement Number U10DD000180, Colorado Department of Public Health/University of Colorado School of Medicine; Cooperative Agreement Number U10DD000181, Kaiser Foundation Research Institute (CA); Cooperative Agreement Number U10DD000182, University of Pennsylvania; Cooperative Agreement Number U10DD000183, Johns Hopkins University; Cooperative Agreement Number U10DD000184, University of North Carolina at Chapel Hill; and Cooperative Agreement Number U10DD000498, Michigan State University. The findings and conclusions in this report are those of the authors and do not necessarily represent the official position of the CDC. Selected, preliminary results were presented at the International Meeting for Autism Research, Baltimore, MD, May 2016, and at the World Conference on Injury Prevention and Safety Promotion, Tampere, Finland, September 2016.

\section{References}

Achenbach, T. Child Behavior Checklist. Burlington, VT: 1992. Vol. Achenbach System of Empirically based Assessment

Allen CW, Silove N, Williams K, Hutchins P. Validity of the social communication questionnaire in assessing risk of autism in preschool children with developmental problems. Journal of Autism and Developmental Disorders. 2007; 37(7):1272-1278. DOI: 10.1007/s10803-006-0279-7 [PubMed: 17080270]

Bijur PE, Kurzon M, Overpeck MD, Scheidt PC. Parental alcohol use, problem drinking, and children's injuries. JAMA. 1992; 267(23):3166-3171. [PubMed: 1593737] 
Borse, NN., Gilchrist, J., Dellinger, AM., Rudd, RA., Ballesteros, MF., Sleet, DA. CDC Childhood Injury Report: Patterns of Unintentional Injuries among 0-19 Year Olds in the United States 2000-2006. 2008. Retrieved from Atlanta (GA): https://www.cdc.gov/safechild/images/CDCchildhoodinjury.pdf

Centers for Disease Control and Prevention. Mental health in the United States: Parental report of diagnosed autism in children aged 4-17 Years-United States, 2003-2004. MMWR. Morbidity and Mortality Weekly News. 2006; 55(17):5. Retrieved from https://www.cdc.gov/mmwr/preview/ mmwrhtml/mm5517a3.htm.

Centers for Disease Control and Prevention. Injury Prevention \& Control: Data \& Statistics (WISQARS $^{\mathrm{TM}}$ ). Dec 22. 2016 Retrieved from http://www.cdc.gov/injury/wisqars/

Chakravarthy B, Anderson CL, Ludlow J, Lotfipour S, Vaca FE. The relationship of pedestrian injuries to socioeconomic characteristics in a large Southern California County. Traffic Injury Prevention. 2010; 11(5):508-513. DOI: 10.1080/15389588.2010.497546 [PubMed: 20872307]

Chen, LH., Warner, M., Fingerhut, L., Makuc, D. Vital and Health Statistics. 2009. Injury episodes and circumstances: National Health Interview Survey, 1997-2007; p. 1-55.Series 10: Data from the National Health Survey

Cummings P, Rivara FP, Thompson RS, Reid RJ. Ability of parents to recall the injuries of their young children. Injury Prevention. 2005; 11(1):43-47. DOI: 10.1136/ip.2004.006833 [PubMed: 15691989]

Daniels JL, Forssen U, Hultman CM, Cnattingius S, Savitz DA, Feychting M, Sparen P. Parental psychiatric disorders associated with autism spectrum disorders in the offspring. Pediatrics. 2008; 121(5):e1357-1362. DOI: 10.1542/peds.2007-2296 [PubMed: 18450879]

DiGuiseppi CG, Daniels JL, Fallin DM, Rosenberg SA, Schieve LA, Thomas KC, Schendel DE. Demographic profile of families and children in the Study to Explore Early Development (SEED): Case-control study of autism spectrum disorder. Disability and Health Journal. 2016; 9(3):544551. DOI: 10.1016/j.dhjo.2016.01.005 [PubMed: 26917104]

Fingerhut LA, Warner M. The ICD-10 injury mortality diagnosis matrix. Injury Prevention. 2006; 12(1):24-29. DOI: 10.1136/ip.2005.009076 [PubMed: 16461416]

Finlayson J, Morrison J, Jackson A, Mantry D, Cooper SA. Injuries, falls and accidents among adults with intellectual disabilities. Prospective cohort study. Journal of Intellectual Disability Research. 2010; 54(11):966-980. DOI: 10.1111/j.1365-2788.2010.01319.x [PubMed: 21040056]

Florence C, Simon T, Haegerich T, Luo F, Zhou C. Estimated lifetime medical and work-loss costs of fatal injuries- United States, 2013. MMWR: Morbidity and Mortality Weekly Report. 2015; 64(38):1074-1077. DOI: 10.15585/mmwr.mm6438a4 [PubMed: 26421530]

Gotham K, Pickles A, Lord C. Standardizing ADOS scores for a measure of severity in autism spectrum disorders. Journal of Autism and Developmental Disorders. 2009; 39(5):693-705. DOI: 10.1007/s10803-008-0674-3 [PubMed: 19082876]

Gotham K, Risi S, Pickles A, Lord C. The Autism Diagnostic Observation Schedule: Revised algorithms for improved diagnostic validity. Journal of Autism and Developmental Disorders. 2007; 37(4):613-627. DOI: 10.1007/s10803-006-0280-1 [PubMed: 17180459]

Guan J, Li G. Injury mortality in individuals with autism. American Journal of Public Health. 2017; doi: 10.2105/AJPH.2017.303696

Haynes R, Reading R, Gale S. Household and neighbourhood risks for injury to 5-14 year old children. Social Science and Medicine. 2003; 57(4):625-636. [PubMed: 12821011]

Hodge D, Hoffman CD, Sweeney DP. Increased psychopathology in parents of children with autism: Genetic liability or burden of caregiving? Journal of Developmental and Physical Disabilities. 2011; 23(3):227-239. DOI: 10.1007/s10882-010-9218-9

Hong J, Lee B, Ha EH, Park H. Parental socioeconomic status and unintentional injury deaths in early childhood: Consideration of injury mechanisms, age at death, and gender. Accident Analysis and Prevention. 2010; 42(1):313-319. DOI: 10.1016/j.aap.2009.08.010 [PubMed: 19887173]

Huang P, Kallan MJ, O’Neil J, Bull MJ, Blum NJ, Durbin DR. Children with special health care needs: Patterns of safety restraint use, seating position, and risk of injury in motor vehicle crashes. Pediatrics. 2009; 123(2):518-523. DOI: 10.1542/peds.2008-0092 [PubMed: 19171617] 
Jain A, Spencer D, Yang W, Kelly JP, Newschaffer CJ, Johnson J, Dennen T. Injuries among children with autism spectrum disorder. Academic Pediatrics. 2014; 14(4):390-397. DOI: 10.1016/j.acap. 2014.03.012 [PubMed: 24976351]

Kalb LG, Vasa RA, Ballard ED, Woods S, Goldstein M, Wilcox HC. Epidemiology of injury-related emergency department visits in the US among youth with autism spectrum disorder. Journal of Autism and Developmental Disorders. 2016; 46(8):2756-2763. DOI: 10.1007/s10803-016-2820-7 [PubMed: 27241347]

Lee LC, David AB, Rusyniak J, Landa R, Newschaffer CJ. Performance of the Social Communication Questionnaire in children receiving preschool special education services. Research in Autism Spectrum Disorders. 2007; 1(2):126-138. DOI: 10.1016/j.rasd.2006.08.004

Lee LC, Harrington RA, Chang JJ, Connors SL. Increased risk of injury in children with developmental disabilities. Research in Developmental Disabilities. 2008; 29(3):247-255. DOI: 10.1016/j.ridd.2007.05.002 [PubMed: 17582739]

Lord, C., Rutter, M., DiLavore, PC., Risi, S. Autism diagnostic observation schedule-WPS (ADOSWPS). Los Angeles, CA: Western Psychological Services; 1999.

Mandell DS, Wiggins LD, Carpenter LA, Daniels J, DiGuiseppi C, Durkin MS, Kirby RS. Racial/ ethnic disparities in the identification of children with autism spectrum disorders. American Journal of Public Health. 2009; 99(3):493-498. DOI: 10.2105/AJPH.2007.131243 [PubMed: 19106426]

McDermott S, Zhou L, Mann J. Injury treatment among children with autism or pervasive developmental disorder. Journal of Autism and Developmental Disorders. 2008; 38(4):626-633. DOI: 10.1007/s10803-007-0426-9 [PubMed: 17690968]

Mullen, E. Mullen scales of early learning. Circle Pines, MN: American Guidance Service, Inc; 1995.

Myhre MC, Thoresen S, Grøgaard JB, Dyb G. Familial factors and child characteristics as predictors of injuries in toddlers: A prospective cohort study. BMJ Open. 2012; 2:e00740.doi: 10.1136/ bmjopen-2011-000740

Mytton J, Towner E, Brussoni M, Gray S. Unintentional injuries in school-aged children and adolescents: Lessons from a systematic review of cohort studies. Injury Prevention. 2009; 15(2): 111-124. DOI: 10.1136/ip.2008.019471 [PubMed: 19346424]

National Center for Health Statistics. ICD-10: External cause of injury mortality matrix [online]. 2002. www.cdc.gov/nchs/injury/injury_matrices.htm

Newschaffer CJ, Croen LA, Daniels J, Giarelli E, Grether JK, Levy SE, Windham GC. The epidemiology of autism spectrum disorders. Annual Review of Public Health. 2007; 28:235-258. DOI: 10.1146/annurev.publhealth.28.021406.144007

Oliver LN, Kohen DE. Neighbourhood variation in hospitalization for unintentional injury among children and teenagers. Health Reports. 2010; 21(4):9-17. [PubMed: 21269007]

Phelan K, Khoury J, Atherton H, Kahn RS. Maternal depression, child behavior, and injury. Injury Prevention. 2007; 13(6):403-408. DOI: 10.1136/ip.2006.014571 [PubMed: 18056318]

Ramirez M, Yang J, Bourque L, Javien J, Kashani S, Limbos MA, Peek-Asa C. Sports injuries to high school athletes with disabilities. Pediatrics. 2009; 123(2):690-696. DOI: 10.1542/peds.2008-0603 [PubMed: 19171639]

Rubenstein E, Pretzel Edmondson, Windham R, Schieve GC, Wiggins LA, DiGuiseppi LDC, Daniels J. The broader autism phenotype in mothers is associated with increased discordance between maternal-reported and clinician-observed instruments that measure child autism spectrum disorder. Journal of Autism and Developmental Disorders. 2017; doi: 10.1007/s10803-017-3248-4

Rutter, ML., Bailey, A., Lord, C. Social communication questionnaire (SCQ): Manual. Western Psychological Services; 2003.

Schendel DE, DiGuiseppi C, Croen LA, Fallin MD, Reed PL, Schieve LA, Yeargin-Allsopp M. The Study to Explore Early Development (SEED): A multisite epidemiologic study of autism by the Centers for Autism and Developmental Disabilities Research and Epidemiology (CADDRE) network. Journal of Autism and Developmental Disorders. 2012; 42(10):2121-2140. DOI: 10.1007/s10803-012-1461-8 [PubMed: 22350336]

Schwebel DC, Brezausek CM. Chronic maternal depression and children's injury risk. Journal of Pediatric Psychology. 2008; 33(10):1108-1116. DOI: 10.1093/jpepsy/jsn046 [PubMed: 18474518] 
Schwebel DC, Gaines J. Pediatric unintentional injury: Behavioral risk factors and implications for prevention. Journal of Developmental and Behavioral Pediatrics. 2007; 28(3):245-254. DOI: 10.1097/01.DBP.0000268561.80204.2a [PubMed: 17565295]

Sikora DM, Vora P, Coury DL, Rosenberg D. Attention-deficit/hyperactivity disorder symptoms, adaptive functioning, and quality of life in children with autism spectrum disorder. Pediatrics. 2012; 130(Suppl 2):S91-97. DOI: 10.1542/peds.2012-0900G [PubMed: 23118259]

Vohra R, Madhavan S, Sambamoorthi U. Emergency department use among adults with autism spectrum disorders (ASD). Journal of Autism and Developmental Disorders. 2016; 46(4):14411454. DOI: 10.1007/s10803-015-2692-2 [PubMed: 26762115]

Wiggins LD, Levy SE, Daniels J, Schieve L, Croen LA, DiGuiseppi C, Schendel D. Autism spectrum disorder symptoms among children enrolled in the Study to Explore Early Development (SEED). Journal of Autism and Developmental Disorders. 2015a; 45(10):3183-3194. DOI: 10.1007/ s10803-015-2476-8 [PubMed: 26048040]

Wiggins LD, Reynolds A, Rice CE, Moody EJ, Bernal P, Blaskey L, Levy SE. Using standardized diagnostic instruments to classify children with autism in the study to explore early development. Journal of Autism and Developmental Disorders. 2015b; 45(5):1271-1280. DOI: 10.1007/ s10803-014-2287-3 [PubMed: 25348175] 


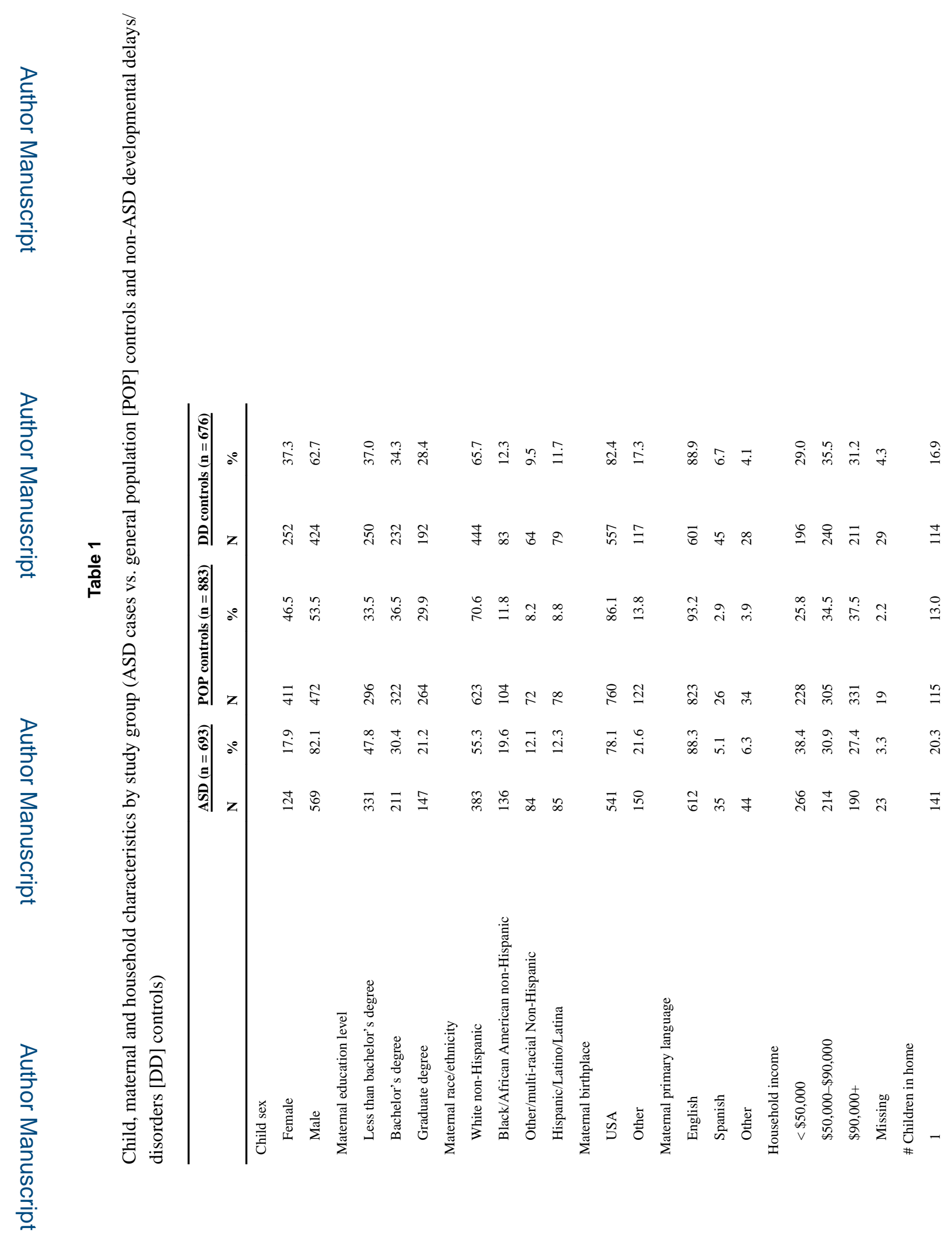

J Autism Dev Disord. Author manuscript; available in PMC 2019 February 01. 
DiGuiseppi et al.

Page 16

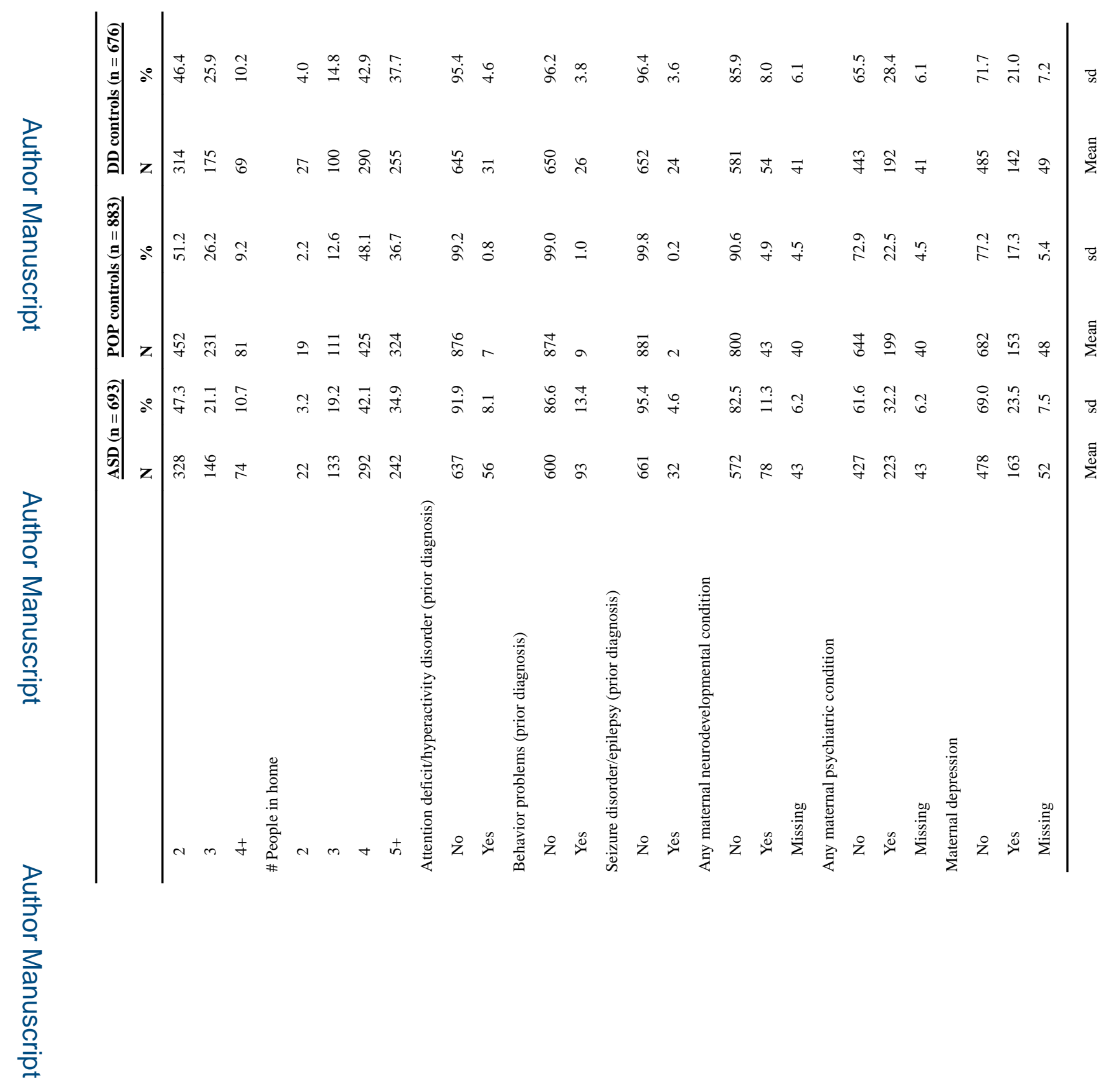

로을

J Autism Dev Disord. Author manuscript; available in PMC 2019 February 01. 
DiGuiseppi et al.

Page 17

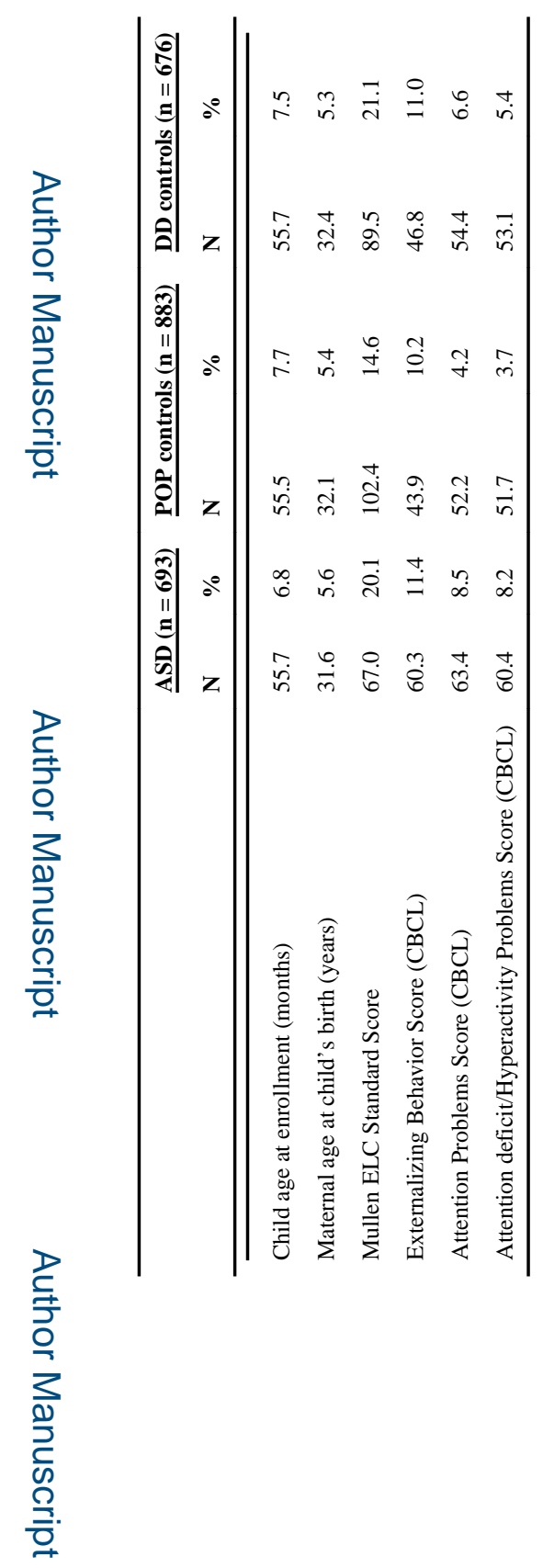

로을

J Autism Dev Disord. Author manuscript; available in PMC 2019 February 01. 


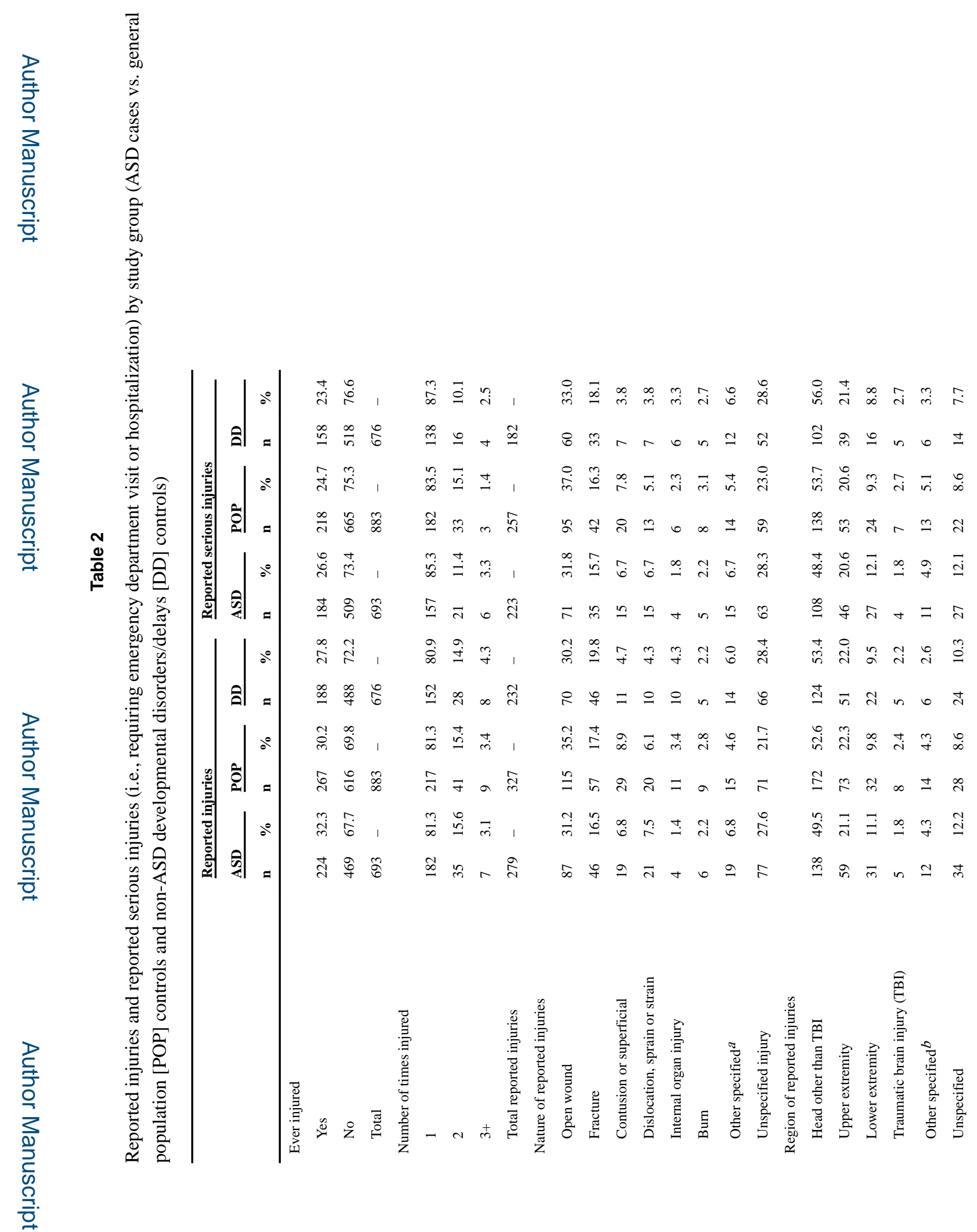

J Autism Dev Disord. Author manuscript; available in PMC 2019 February 01. 


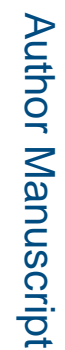

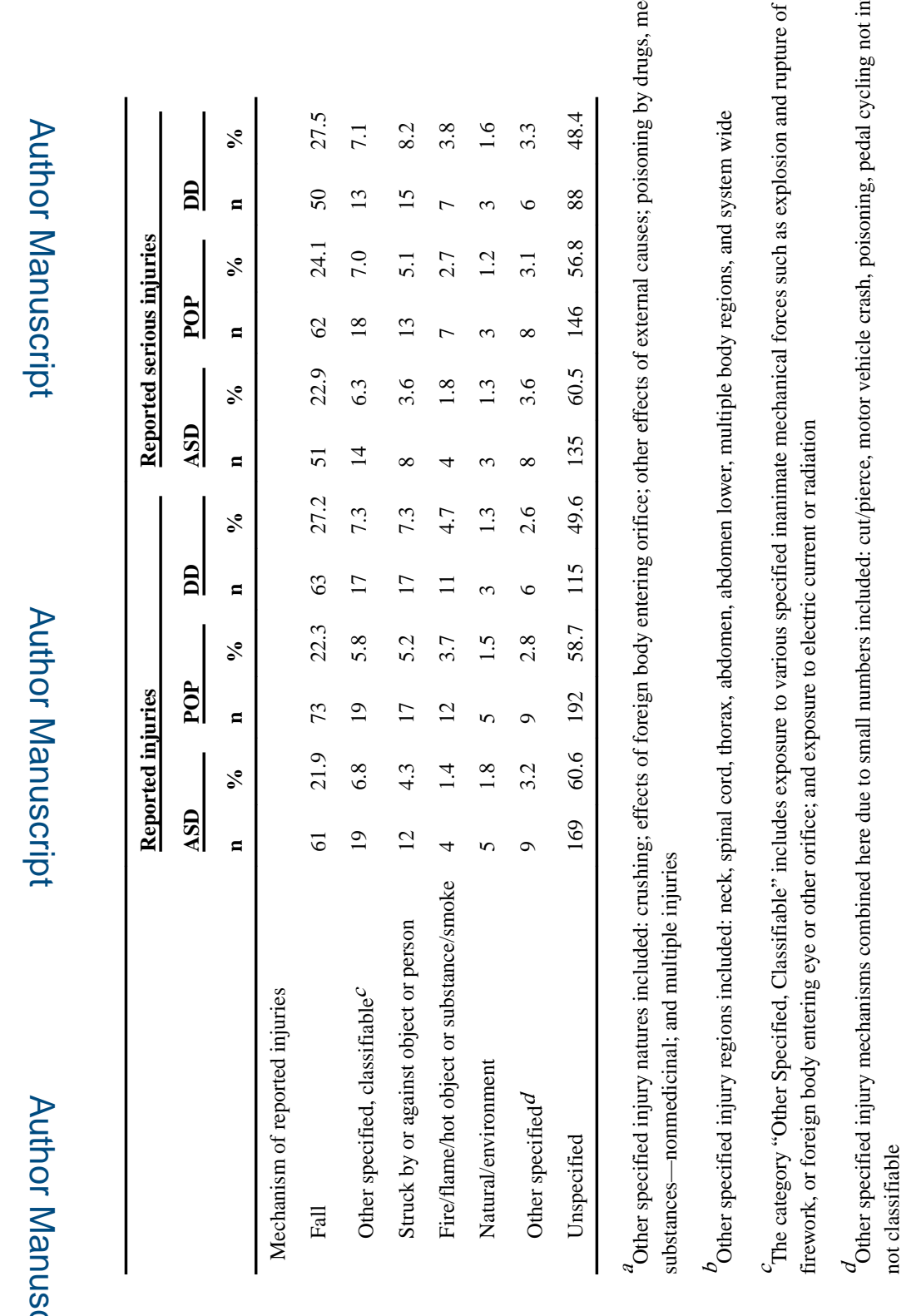




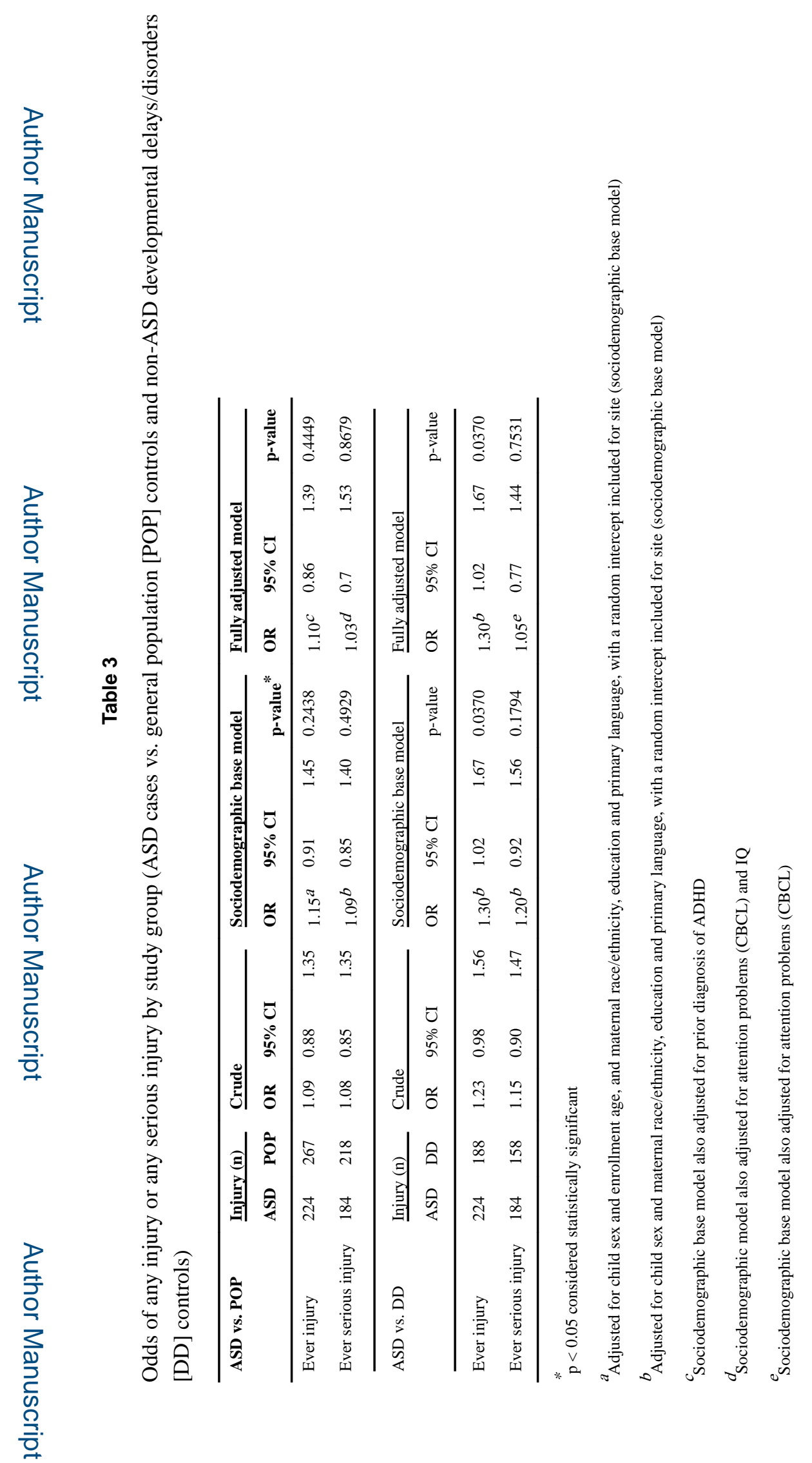

J Autism Dev Disord. Author manuscript; available in PMC 2019 February 01. 